\title{
Theoretical Analysis of Five-Point Bending and Springback for Preforming Process of ERW Pipe FFX Forming
}

\author{
Lidong Ma ${ }^{\mathbb{D}},{ }^{1,2}$ Haoxi Ma, ${ }^{1}$ Zijian Liu, ${ }^{1}$ and Shuo Chen ${ }^{1}$ \\ ${ }^{1}$ Coordinative Innovation Center of Taiyuan Heavy Machinery Equipment, Taiyuan University of Science and Technology, \\ Taiyuan 030024, China \\ ${ }^{2}$ Key Laboratory of Metallurgical Equipment Design Theory and Technology of Shanxi Province, \\ Taiyuan University of Science and Technology, Taiyuan 030024, China \\ Correspondence should be addressed to Lidong Ma; mald@tyust.edu.cn
}

Received 3 March 2019; Revised 29 April 2019; Accepted 13 May 2019; Published 23 June 2019

Academic Editor: Hervé Laurent

Copyright (C) 2019 Lidong Ma et al. This is an open access article distributed under the Creative Commons Attribution License, which permits unrestricted use, distribution, and reproduction in any medium, provided the original work is properly cited.

Flexible forming excellent (FFX) forming of electric resistance welding (ERW) pipe is an advanced roll forming method. In order to understand FFX forming mechanism and set reasonable roll parameters, five-point bending process and springback process for preforming section of ERW pipe FFX forming are analyzed. Under various assumptions, the bending process of sheet metal is divided into full elastic bending stage and elastic-plastic bending stage by analyzing the forming law and simplifying the contact state model of roll bending process. Based on the theory of plane bending theory, the five-point bending springback model is established. Mathematical expressions of bending curvature and rotation angle of arbitrary particle on plate under arbitrary state are derived. The curvature calculation formulas of each particle on plate after springback are obtained, and then the calculation formulas of bending angle and rebound angle after springback are obtained. The theoretical analysis results are verified by the wide plate five-point bending experiment. The theoretical value of springback angle agrees well with the experimental results. The maximum deviation of springback angle is 0.42 degrees. The results show that the five-point bending model is reasonable. It provides theoretical support for the preforming model of flexible roll forming.

\section{Introduction}

In recent years, ERW pipe products have caused extensive concern [1, 2]. High-grade ERW pipes are mainly used for oil wells, submarine pipelines, oil and gas pipelines, some special purpose professional pipes, etc. The forming process for producing high-grade ERW pipes is mainly divided into two types: the first one is pressure forming, such as JCO forming and UOE forming. The second one is roll forming, including row roll forming, cage roll forming, and other flexible roll forming methods. Roll forming is cost-effective compared with other sheet metal forming processes. Flexible roll forming can use the same unit to form a certain range of products and become more and more flexible. The motion of the rolls can be controlled by many axes enabling a large variation in the profiles along the formed sheet, called 3D roll forming or flexible roll forming technology [3]. Nowadays, flexible roll forming technology has been applied in many fields. Zhong [4] researched the flexible roll forming process and established carbon fiber composite process control software. Joo [5] proposed a profile design method for manufacturing variable section parts by flexible forming process. Kari [6] proved that UHS steel can be bent by flexible roll forming process through experimental research. And flexible roll forming technology has also been widely used in the manufacture of automotive component, hull structure, and three-dimensional surface parts [7-9]. Nakata Flexible forming excellent (FFX) forming is an advanced flexible roll forming technology [10, 11].

Multipoint bending forming is a popular forming method and a flexible manufacturing technique. It is especially suited to the three-dimensional forming of varied large sheet [12]. The five-point bending forming proposed in the paper is a multipoint bending forming technology, it is the forming method for the preforming section of the FFX forming process, and the middle of the sheet metal is in a state of reverse 
bending deformation called W-forming, which makes the edge bending deformation sufficient and the amount of springback small. In actual production, this forming process can reduce the defects such as bad edge bending caused by material properties fluctuation and roll wear.

Springback is the elastically driven change during unloading and following forming [13]. In the past twenty years, more and more researchers began to pay attention to and study this topic, and this topic has developed rapidly. Carden et al. [14] measured the springback by stretching and bending experiments. Chen [15] and Wagoner [16] carried out finite element simulation of springback of sheet metal forming by different methods. Zhao et al. [17] established the complex equation of planar bending elastic and successfully applied it to the expanding and setting round process. On this basis, Zhao et al. also carried out some research on the prediction and control of the springback in setting round process for pipe-end of large pipe and the springback analysis of profile plane stretch-bending in the loading method of pretension and moment [18, 19]. Liu et al. [20] proposed an analytical method for predicting springback and residual stress distribution of thick plates after multipoint forming. Bending springback is one of the most important factors in ERW pipe forming process. At present, few experts research on bending springback of FFX forming technology, so it cannot provide theoretical guidance for engineering practice. The preforming section of FFX forming is a key process affecting the final product quality. Therefore, it is of great significance to carry out theoretical research on the bending springback process of the FFX forming preformed section.

Based on the classical elastic-plastic bending theory and the analytical theory of three-point bending and fourpoint bending of JCO welded pipe forming [21, 22], the in-depth study of the bending and springback process of the preforming process is carried out, and the mechanical model of the five-point bending springback of the plate is established. It lays a theoretical foundation for realizing the prediction model of roll position parameters of the preformed section in FFX intelligent control system of ERW pipe.

\section{Basic Theory of Bending Springback in Plate Forming Process}

2.1. Basic Assumptions. (1) Flat section assumption means that the cross section of the plate is always perpendicular to the axis of the plate during deformation before and after.

(2) Tension-free assumption means that the sheet has no longitudinal stress during the bending process.

(3) During the bending process, the stress neutral layer, the strain neutral layer, and the geometric center layer of the plate always coincide.

(4) The deformation area conforms to the assumption of small deformation, which means the strain distribution on the section is linear distribution, and the expression is as follows:

$$
\varepsilon=\frac{y}{\rho}
$$

where $\varepsilon$ is the strain; $\rho$ is the radius of curvature of neutral layer; $y$ is the distance from the mass point to the neutral layer on the section.

(5) The material conforms to the exponential hardening model, and the expression is as follows:

$$
\begin{array}{cc}
\sigma=E \varepsilon & \varepsilon \leq \frac{\sigma_{s}}{E} \\
\sigma=k \varepsilon^{n} & \varepsilon>\frac{\sigma_{s}}{E}
\end{array}
$$

where $\sigma$ is the stress; $k$ is the coefficient of strength; $n$ is the hardening index; $\sigma_{s}$ is the material yield limit; $E$ is the modulus of elasticity.

2.2. Analysis of Preforming Process of FFX Forming. The springback process is analyzed, as shown in Figure 1 (Figure 1 shows the forming part of the first rack of preforming section in FFX forming mode). The bending state of the plate is analyzed, and different roll gaps correspond to different bending directions.

At present, the specific roll profile data of FFX forming mode is not considered, it is simplified appropriately in the derivation process, and its deformation state is similar to Figure 2. The bending process is divided into elastic bending stage and elastic-plastic bending stage according to whether the upper roller has been coated. Figure 3 shows the bending moment under different coating states (the bending moment state in actual forming process can be adjusted based on this model). There are two quantities involved, $\rho_{\text {lim }}$ and $\rho_{\min }$, where $\rho_{\min }$ is the minimum bending radius in the deformation process and $\rho_{\text {lim }}$ is the elastic limit radius. The calculation formula is as follows:

$$
\rho_{\text {lim }}=\frac{E t}{2 \sigma_{s}}
$$

Taking the coating state of plate and upper roller as the boundary point, the bending process of plate is divided into two stages. The first is that the plate begins to contact the upper roller. The second is that the plate coats the upper roller. In the first stage, the bending starts and the contact state between plate and five rollers is point contact, then the bending state changes into a five-point bending state. All particles in the wide plate may be in the elastic-plastic stage, and the distribution of bending moment is shown in Figure 3(a). In the second stage, the plate begins to coat the upper roller until it is completely coated. At this time, the plate has entered the stage of elastic-plastic deformation, and the bending moment in the coating area reaches the maximum value. The distribution of bending moment is shown in Figure 3(b). 

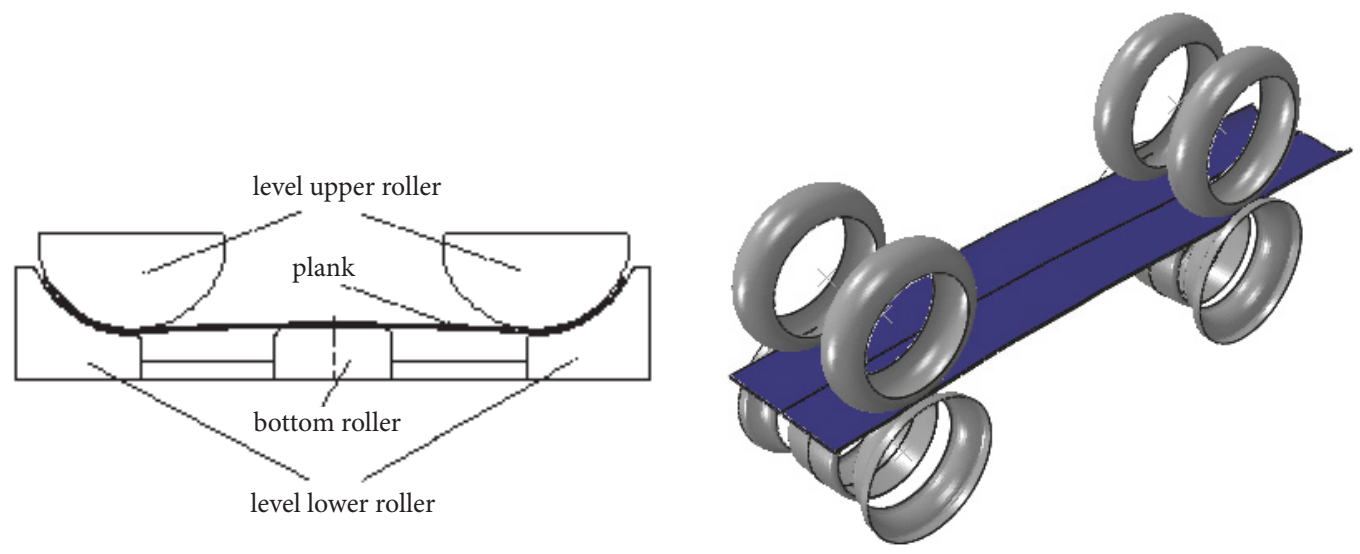

FIGURE 1: Analysis of contact state of roll bending deformation process.

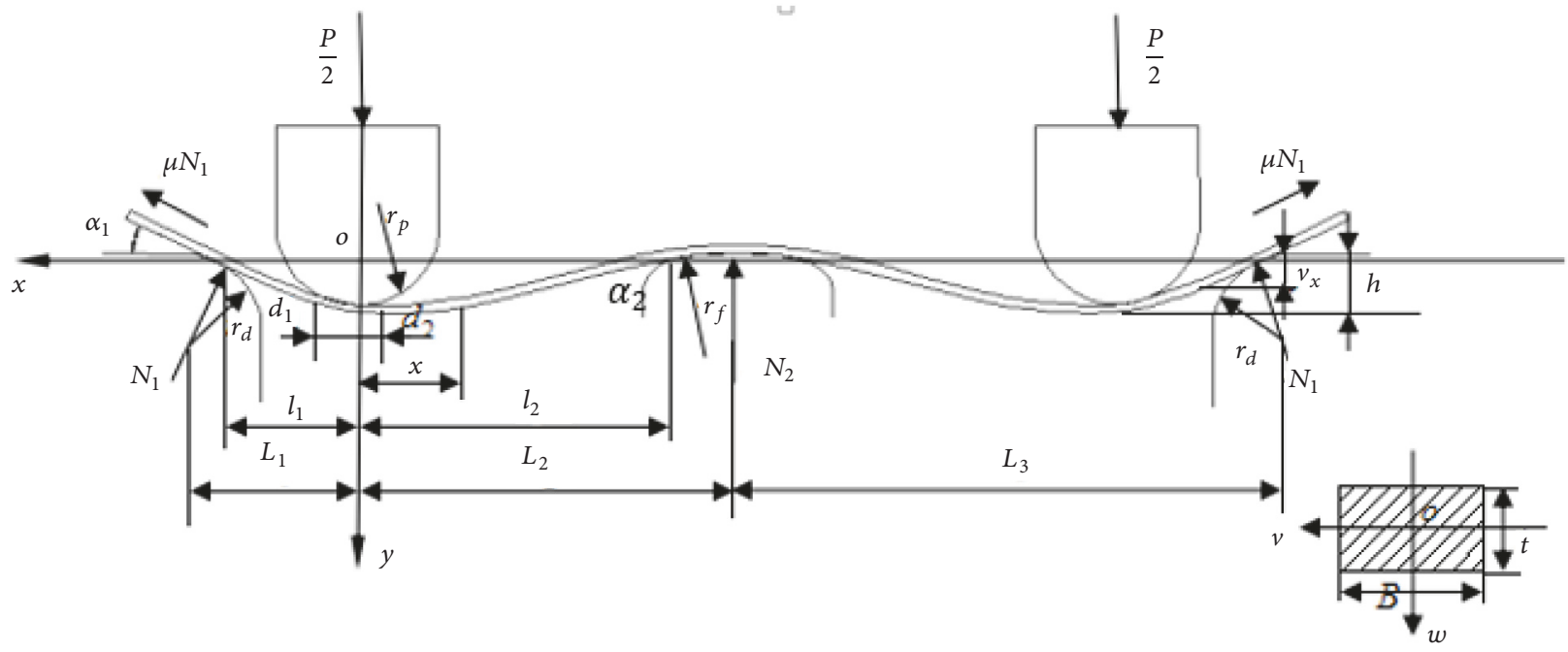

FIGURE 2: Broad plate five-point bending diagram.

obtained from the static equilibrium equation and the geometric relationship:

$$
\begin{aligned}
& P=2 N_{1}\left(\cos \alpha_{1}+\mu \sin \alpha_{1}\right)+N_{2} \\
& l_{1}=L_{1}-\left(r_{d}+\frac{t}{2}\right) \sin \alpha_{1} \\
& l_{2}=L_{2}-\left(r_{f}+\frac{t}{2}\right) \sin \alpha_{2}
\end{aligned}
$$

where $P$ is the bending force; $\mu$ is the friction coefficient; $t$ is the thickness; $r_{d}$ and $r_{f}$ are the rounded corner of the level lower roller and the bottom roller, respectively; $N_{1}$ and $N_{2}$ are the reaction forces at the fulcrum of the lower roller and the bottom roller respectively; $\alpha_{1}$ and $\alpha_{2}$ are the corner of the fulcrum of the lower roller and the bottom roller respectively; $l_{1}$ and $l_{2}$ are the distance from the origin of coordinates to the cutting point of the lower roller and the bottom roller; $L_{1}$ and $L_{2}$ are the distance from the origin of coordinates to the center of the lower roller corner and the center of the bottom roller corner, respectively.

\section{Full Elastic Bending Stage}

When the upper roller is pressed down, the initial stage of contact between the strip and the upper roller is the full elastic bending stage. The full elastic bending stage refers to the fact that the minimum bending radius $\rho_{\min }$ of each point on the neutral layer of the plate belt is always greater than the elastic limit bending radius $\rho_{\text {lim }}$. In this state, the strip will rebound after the external force is removed.

At this stage, the bending moment acting on the strip presents a linear distribution. Define the bending moment of the strip section at the distance from origin 0 to $x$ as $M_{x}$, then 


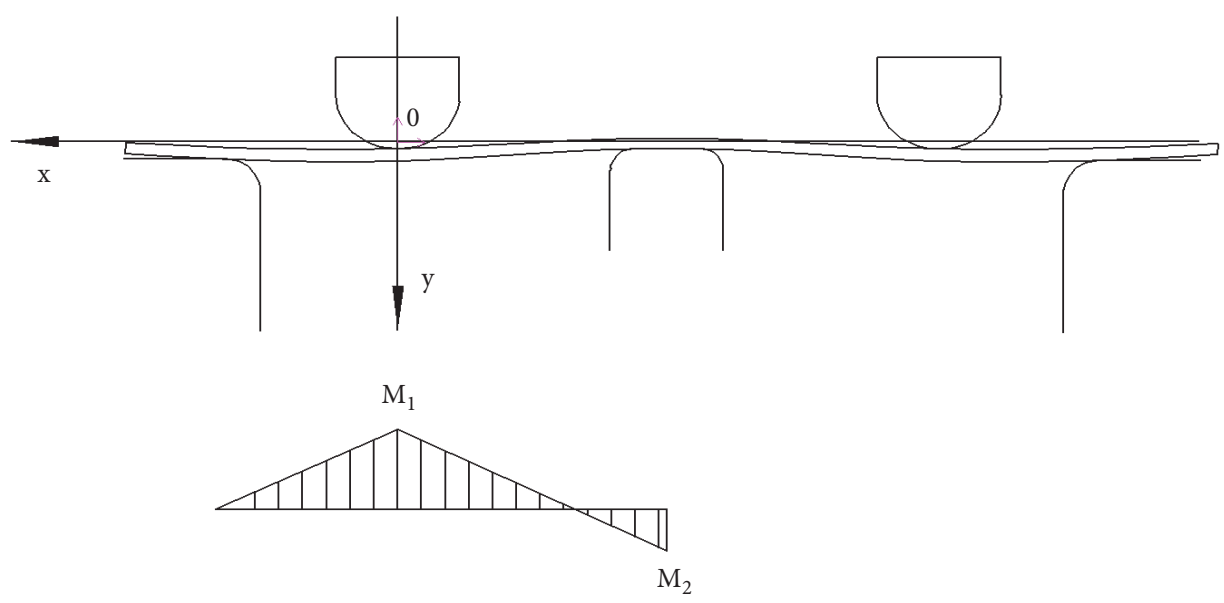

(a) Upper roller uncoating stage
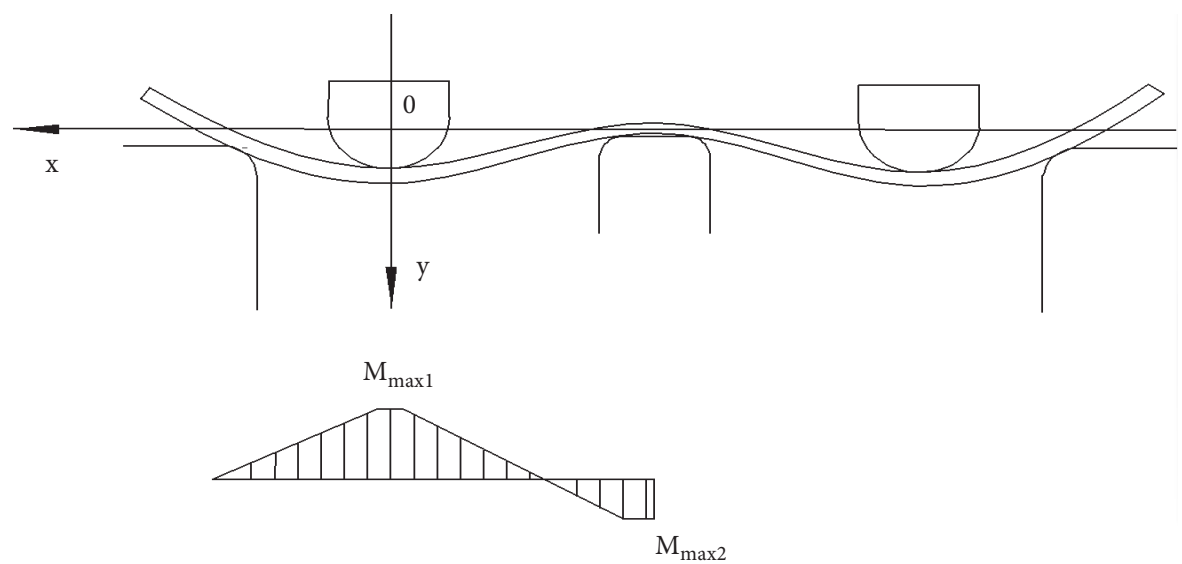

(b) Upper roller coating stage

FIGURE 3: Bending process and the distribution of bending moment.

$$
M_{x}= \begin{cases}N_{1} l_{1}\left(1-\frac{x}{l_{1}}\right) \frac{1}{\cos \alpha_{1}} & x \in\left[0, l_{1}\right] \\ N_{1} l_{1}\left(1+\frac{x}{l_{2}}\right) \frac{1}{\cos \alpha_{1}}+\frac{N_{2} x}{2} & x \in\left[-l_{2}, 0\right)\end{cases}
$$

According to the knowledge of material mechanics, the relation between bending moment and radius of curvature is

$$
\frac{1}{\rho_{x}}=\frac{M_{x}}{E I}
$$

where $\rho_{x}$ is the radius of curvature at any point $x$ on the neutral layer.

From (7) and (8) we can obtain

$$
\frac{1}{\rho_{x}}= \begin{cases}\frac{N_{1} l_{1}}{E I}\left(1-\frac{x}{l_{1}}\right) \frac{1}{\cos \alpha_{1}} & x \in\left[0, l_{1}\right] \\ \frac{N_{1} l_{1}}{E I}\left(1+\frac{x}{l_{2}}\right) \frac{1}{\cos \alpha_{1}}+\frac{N_{2} x}{2} & x \in\left[-l_{2}, 0\right)\end{cases}
$$

Define the curvature of the strip at any point $x$ as $K_{x}\left(K_{x} \geq\right.$ $0)$, then

$$
K_{x}=\frac{\left|y^{\prime \prime}(x)\right|}{\left[1+y^{\prime 2}(x)\right]^{3 / 2}}
$$

Let $d y / d x=\tan u$, then $d^{2} y / d x^{2}=\left(1 / \cos ^{2} u\right)(d u / d x)$. Because of $K_{x} \geq 0$, formula (10) can be simplified as

$$
K_{x}=-\cos u \frac{d u}{d x}
$$

For $K_{x}=1 / \rho_{x}$, we can get

$$
-\cos u \frac{d u}{d x}
$$

$$
= \begin{cases}\frac{N_{1} l_{1}}{E I}\left(1-\frac{x}{l_{1}}\right) \frac{1}{\cos \alpha_{1}} & x \in\left[0, l_{1}\right] \\ \frac{N_{1} l_{1}}{E I}\left(1+\frac{x}{l_{2}}\right) \frac{1}{\cos \alpha_{1}}+\frac{N_{2} x}{2} & x \in\left[-l_{2}, 0\right)\end{cases}
$$


Define the angle of rotation at the particle $x$ as $\alpha_{x}$, then $\alpha_{x}=$ $u$. Thus,

$$
\begin{aligned}
& \alpha_{x}=\sin ^{-1} f(x, p) \\
& f(x, p) \\
& = \begin{cases}-\frac{N_{1} l_{1}}{E I \cos \alpha_{1}}\left(x-\frac{x^{2}}{2 l_{1}}\right), & x \in\left[0, l_{1}\right] \\
-\frac{N_{1} l_{1}}{E I \cos \alpha_{1}}\left(x-\frac{x^{2}}{2 l_{2}}\right)-\frac{N_{2} x}{4}, & x \in\left[-l_{2}, 0\right)\end{cases}
\end{aligned}
$$

According to the boundary condition $\left.y\right|_{x=l_{1}}=0$, the deflection $y_{x}$ at any point in $x \in\left[0, l_{1}\right]$ is

$$
y_{x}=\int_{0}^{x} \frac{f(x, p)}{\sqrt{1-f^{2}(x, p)}} d x-\int_{0}^{l_{1}} \frac{f(x, p)}{\sqrt{1-f^{2}(x, p)}} d x
$$

The displacement $h$ of the upper roller is

$$
h=y_{\max }+\left(r_{d}+\frac{t}{2}\right)\left(1-\cos \alpha_{1}\right)
$$

Since any particle in the steel plate is in an elastic deformation state, it rebound completely.

\section{Elastic-Plastic Bending Stage}

4.1. Upper Roller Uncoating Stage. At this stage, plastic deformation of a particle in the strip has occurred. The minimum bending radius $\rho_{\min }$ is greater than the circular radius $r_{p}$ of the upper roller and less than the elastic limit bending radius, that is, $r_{p}<\rho_{\min }<\rho_{\text {lim }}$.

(1) The Bending Process. According to the theory of wide plate free bending forming [23], another expression of the bending moment at the section $x$ of the strip can be obtained.

$$
M_{x}=\int_{A} \sigma w d A=\frac{k I_{p}}{\rho_{x}^{n}}
$$

where $k$ is strengthening coefficient; $n$ is hardening index; $I_{p}$ is moment of inertia of plastic bending section of exponential hardening material, $I_{p}=\int_{A} w^{n+1} d A$.

From (8), (10), and (11), we have

$$
\frac{1}{\rho_{x}}=\left[\frac{M_{x}}{k I_{p}}\right]^{1 / n}
$$

Define the angle of rotation at the particle $x$ as $\alpha_{x}$, then $\alpha_{x}=u$; therefore,

$$
\begin{aligned}
& \alpha_{x}=\sin ^{-1} f(x, p) \\
& f(x, p)=-\frac{n}{n+1}\left(\frac{N_{1} l_{1}}{k I_{p} \cos \alpha_{1}}\right)^{1 / n} l_{1}[1 \\
& \left.-\left(1-\frac{x}{l_{1}}\right)^{(1+n) / n}\right] \quad x \in\left[0, l_{1}\right] \\
& f(x, p)=\frac{n}{n+1}\left[\left(\frac{N_{1} l_{1}}{k I_{p} \cos \alpha_{1}}\right)^{(1+n) / n}\right. \\
& \left.-\left(\frac{N_{1} l_{1}}{k I_{p} \cos \alpha_{1}}\left(1+\frac{x}{l_{2}}\right)+\frac{N_{2} x}{2 k I_{p}}\right)^{(1+n) / n}\right] \\
& \cdot \frac{k I_{p} \cos \alpha_{1}}{N_{1}+\left(N_{2} / 2\right) \cos \alpha_{1}} \quad x \in\left[-l_{2}, 0\right)
\end{aligned}
$$

According to the boundary condition $\left.y\right|_{x=l_{1}}=0$, the deflection $y_{x}$ of particle $x$ is

$$
\begin{array}{r}
y_{x}=\int_{0}^{x} \frac{f(x, p)}{\sqrt{1-f^{2}(x, p)}} d x-\int_{0}^{l_{1}} \frac{f(x, p)}{\sqrt{1-f^{2}(x, p)}} d x \\
x \in\left[0, l_{1}\right]
\end{array}
$$

The displacement $h$ of the upper roller is

$$
h=y_{\max }+\left(r_{d}+\frac{t}{2}\right)\left(1-\cos \alpha_{1}\right)
$$

(2) The Springback Process. When $x=0$, the minimum bending radius is

$$
\rho_{\min }=\left(\frac{k I_{p} \cos \alpha_{1}}{N_{1} l_{1}}\right)^{1 / n}
$$

Let $x_{s 1}$ be the dividing point of the elastic-plastic deformation of the strip in the positive direction of $x$, then $\rho_{x_{s 1}}=$ $\rho_{\text {lim. }}$. Combining (3) and (23), we get

$$
x_{s 1}=l_{1}-\left(\frac{2 \sigma_{s}}{E t}\right)^{n} \frac{k I_{p} \cos \alpha_{1}}{N_{1}}
$$

Let $x_{s 2}$ be the dividing point of the elastic-plastic deformation of the strip in the negative direction of $x$, then $\rho_{x_{s 2}}=$ $\rho_{\text {lim }}$. Combining (3) and (23), we get

$$
x_{s 2}=l_{0} \cdot\left(1-\frac{2 \sigma_{s} I \cos \alpha_{1}}{N_{1} l_{1}}\right)
$$

where $l_{0}$ is the distance from the point in $x \in\left[-l_{2},-d_{2}\right]$ whose bending moment is zero to the original point. And $l_{0}=\left(2 N_{1} l_{1}^{2}-N_{2} l_{2}^{2} \cos \alpha_{1}\right) /\left(2 N_{1} l_{1}-N_{2} l_{2} \cos \alpha_{1}\right)$.

Define the radius of curvature at the point $x$ after springback as $\rho_{x}^{u}$. According to unloading law, we have

$$
\frac{1}{\rho_{x}^{u}}=\frac{1}{\rho_{x}}-\frac{1}{\rho_{e}}
$$



then

Define the bending moment of elastic deformation as $M_{e}$,

$$
M_{e}=\frac{E I}{\rho_{e}}
$$

According to unloading law, the springback bending moment is equal to the bending moment at loading, that is, $M_{x}=M_{e}$. The radius of curvature at particle $x$ after rebound is

$$
\begin{aligned}
\frac{1}{\rho_{x}^{u}}= & {\left[\frac{N_{1} l_{1}}{k I_{p} \cos \alpha_{1}}\left(1-\frac{x}{l_{1}}\right)\right]^{1 / n} } \\
& -\frac{N_{1} l_{1}}{E I \cos \alpha_{1}}\left(1-\frac{x}{l_{1}}\right) \quad x \in\left[0, l_{1}\right] \\
\frac{1}{\rho_{x}^{u}}= & {\left[\frac{N_{1} l_{1}}{k I_{p} \cos \alpha_{1}}\left(1+\frac{x}{l_{2}}\right)+\frac{N_{2} x}{2 k I_{p}}\right]^{1 / n} } \\
& -\frac{N_{1} l_{1}}{E I \cos \alpha_{1}}\left(1+\frac{x}{l_{2}}\right)-\frac{N_{2} x}{2 E I} \quad x \in\left[-l_{2}, 0\right)
\end{aligned}
$$

The rotation angle $\alpha_{x}^{u}$ at particle $x$ after springback is

$$
\alpha_{x}^{u}=\sin ^{-1} g(x, p)
$$

where

$$
\begin{gathered}
g(x, p)=-\frac{n}{n+1}\left(\frac{N_{1} l_{1}}{k I_{p} \cos \alpha_{1}}\right)^{1 / n} l_{1}[1 \\
\left.-\left(1-\frac{x}{l_{1}}\right)^{(n+1) / n}\right]+\frac{N_{1} l_{1}}{E I \cos \alpha_{1}}\left(x-\frac{x^{2}}{2 l_{1}}\right) \\
g(x, p)=\frac{n}{n+1}\left[\left(\frac{N_{1} l_{1}}{k I_{p} \cos \alpha_{1}}\right)^{(1+n) / n} \quad x, l_{1}\right] \\
\left.-\left(\frac{N_{1} l_{1}}{k I_{p} \cos \alpha_{1}}\left(1+\frac{x}{l_{2}}\right)+\frac{N_{2} x}{2 k I_{p}}\right)^{(1+n) / n}\right] \\
\cdot \frac{k I_{p} \cos \alpha_{1}}{N_{1}+P \cos \alpha_{1}}+\frac{N_{1} l_{1}\left(x^{2} / 2 l_{2}+x\right)}{E I_{p} \cos \alpha_{1}}+\frac{N_{2} x^{2}}{4 E I} \\
x \in\left[-l_{2}, 0\right)
\end{gathered}
$$

4.2. Upper Roller Coating Stage. As the upper roller continues to press down, the bending degree of the plate increases, and the plate begins to coat the bottom roller until it is completely coated. At this point, the bending moment distribution is shown in Figure 3(b). The bending moment at the section of the strip is

$$
\begin{aligned}
& M_{x} \\
& = \begin{cases}M_{\max 1}, & x \in\left[-d_{2}, d_{1}\right] \\
M_{\max 1} \cdot \frac{l_{1}-x}{l_{1}-d_{1}}, & x \in\left(d_{1}, l_{1}\right] \\
\frac{M_{\max 1}\left(l_{2}+x\right)-M_{\max 2}\left(d_{2}+x\right)}{l_{2}-d_{2}}, & x \in\left[-l_{2},-d_{2}\right) \\
M_{\max 2}, & x \in\left[-L_{2},-l_{2}\right)\end{cases}
\end{aligned}
$$

According to (16), the maximum bending moment is

$$
\begin{aligned}
& M_{\max 1}=\frac{k I_{p}}{\left(r_{p}+t / 2\right)^{n}} \\
& M_{\max 2}=-\frac{k I_{p}}{\left(r_{f}+t / 2\right)^{n}}
\end{aligned}
$$

According to the mechanical and geometric relations, the bending moment loaded on the $x$ section is

$$
\begin{aligned}
& M_{x} \\
& = \begin{cases}N_{1} l_{1}\left(1-\frac{d_{1}}{l_{1}}\right) \frac{1}{\cos \alpha_{1}}, & x \in\left[-d_{2}, d_{1}\right] \\
N_{1} l_{1}\left(1-\frac{x}{l_{1}}\right) \frac{1}{\cos \alpha_{1}}, & x \in\left[d_{1}, l_{1}\right] \\
\frac{N_{1}\left(l_{1}-d_{1}\right)}{\cos \alpha_{1}} \cdot \frac{l_{2}+x}{l_{2}-d_{2}}+\frac{N_{2}\left(x+d_{2}\right)}{2}, & x \in\left[-l_{2},-d_{2}\right)\end{cases}
\end{aligned}
$$

From (33), (34), (35), and (36), we obtain

$$
\begin{aligned}
& d_{1}=l_{1}-\frac{k I_{p} \cos \alpha_{1}}{N_{1}\left(r_{p}+t / 2\right)^{n}} \\
& d_{2}=l_{2}+\frac{2 k I_{p}}{N_{2}\left(r_{f}+t / 2\right)^{n}}
\end{aligned}
$$

The following discussion is based on the distribution of bending moment.

Case 1. The bending moment is a fixed value in $\left[-d_{2}, d_{1}\right]$.

(1) The Bending Process. According to the geometric relationship in Figure 2, the deflection of particle $x$ in the bending process is

$$
y_{x}=y_{\max }-\left(\left(r_{p}+\frac{t}{2}\right)-\sqrt{\left(r_{p}+\frac{t}{2}\right)^{2}-x^{2}}\right)
$$

The angle of rotation at particle $x$ is

$$
\alpha_{x}=-\sin ^{-1}\left(\frac{x}{r_{p}+t / 2}\right)
$$

(2) The Springback Process. According to the unloading law, the radius of curvature at the particle $x$ after springback is

$$
\frac{1}{\rho_{x}^{u}}=\frac{1}{r_{p}+t / 2}-\frac{M_{\max }}{E I}
$$


The rotation angle of particle $x$ after unloading and springback is

$$
a_{x}^{u}=\sin ^{-1}\left[x\left(\frac{1}{r_{p}+t / 2}-\frac{M_{\max }}{E I}\right)\right]
$$

Case 2. Bending moment is in a linear distribution area $\left(d_{1}, l_{1}\right]$.

(1) The Bending Process. According to Figure 3, the particle in $\left[d_{1}, x_{t 1}\right]$ is in an elastic-plastic state, and the particle in $\left(x_{t 1}, l_{1}\right]$ is in a fully elastic state. Then the radius of curvature at point $x$ is

$$
\frac{1}{\rho_{x}}=\left[\frac{\left(l_{1}-x\right) M_{\max 1}}{k I_{p}\left(l_{1}-d_{1}\right)}\right]^{1 / n} \quad x \in\left[d_{1}, l_{1}\right]
$$

When $x=x_{t 2}, \rho_{x_{t 2}}=\rho_{\text {lim }}$, the boundary point $x_{t 1}$ of elastic-plastic deformation can be obtained from (43).

$$
x_{t 1}=l_{1}-\left(\frac{2 \sigma_{s}}{E t}\right)^{n} \frac{k I_{p}\left(l_{1}-d_{1}\right)}{M_{\max 1}}
$$

Then the angle of rotation at particle $x$ is

$$
\begin{aligned}
& \alpha_{x}=\sin ^{-1} \varphi_{1}(x) \quad x \in\left[d_{1}, l_{1}\right] \\
& \varphi_{1}(x)=\frac{n}{n+1}\left[\frac{M_{\max 1}}{k I_{p}\left(l_{1}-d_{1}\right)}\right]^{1 / n} \\
& \cdot\left[\left(l_{1}-x\right)^{(1+n) / n}-\left(l_{1}-d_{1}\right)^{(1+n) / n}\right]-\frac{d_{1}}{r_{p}+t / 2}
\end{aligned}
$$

According to the boundary conditions $\left.y\right|_{x=l}=0$, the deflection at the particle $x$ is

$$
y_{x}=\int_{d_{1}}^{x} \frac{\varphi_{1}(x)}{\sqrt{1-\varphi_{1}^{2}(x)}} d x-\int_{d_{1}}^{l_{1}} \frac{\varphi_{1}(x)}{\sqrt{1-\varphi_{1}^{2}(x)}} d x
$$

The upper roller stroke is

$$
\begin{aligned}
h= & y_{\max }+\left(r_{d}+\frac{t}{2}\right)\left(1-\cos \alpha_{1}\right)+r_{d}+\frac{t}{2} \\
& -\sqrt{\left(r_{d}+\frac{t}{2}\right)^{2}-d_{1}{ }^{2}}
\end{aligned}
$$

(2) The Springback Process. According to the unloading law, the radius of curvature at particle $x$ after springback is

$$
\begin{array}{r}
\frac{1}{\rho_{x}^{u}}=\left[\frac{M_{\max 1}\left(l_{1}-x\right)}{k I_{p}\left(l_{1}-d_{1}\right)}\right]^{1 / n}-\frac{M_{\max 1}}{E I} \frac{\left(l_{1}-x\right)}{\left(l_{1}-d_{1}\right)} \\
x \in\left[d_{1}, x_{t 1}\right]
\end{array}
$$

The rotation angle at particle $x \in\left[d_{1}, x_{t 1}\right]$ after unloading and springback is

$$
\begin{aligned}
& \alpha_{x}^{u}=\sin ^{-1} \phi_{1}(x) \\
& \phi_{1}(x)=\frac{n}{n+1}\left[\frac{M_{\max 1}}{k I_{p}\left(l_{1}-d_{1}\right)}\right]^{1 / n} \\
& \cdot\left[\left(l_{1}-x\right)^{(n+1) / n}-\left(l_{1}-d_{1}\right)^{(n+1) / n}\right] \\
& -\frac{M_{\max 1}}{2 E I\left(l_{1}-d_{1}\right)}\left[\left(l_{1}-x\right)^{2}-\left(l_{1}-d_{1}\right)^{2}\right] \\
& -d_{1}\left(\frac{1}{r_{p}+t / 2}-\frac{M_{\max 1}}{E I}\right)
\end{aligned}
$$

Case 3. Bending moment is in a linear distribution area $\left[-l_{2},-d_{2}\right]$.

(1) The Bending Process. According to Figure 3, the particle in $\left[-l_{2}, x_{t 3}\right] \cup\left[x_{t 2},-d_{2}\right]$ is in an elastic-plastic state, and the particle in $\left(x_{t 2}, x_{t 3}\right)$ is in a fully elastic state.

The radius of curvature at particle $x$ is

$$
\frac{1}{\rho_{x}}=\left[\frac{\left(l_{0}+x\right) M_{\max 1}}{k I_{p}\left(l_{0}-d_{2}\right)}\right]^{1 / n} \quad x \in\left[-x_{t 2},-d_{2}\right]
$$

where $l_{0}$ is the distance from the point in $x \in\left[-l_{2},-d_{2}\right]$ whose bending moment is zero to the origin point. And $l_{0}=$ $\left(M_{\max 1} l_{1}-M_{\max 2} l_{2}\right) /\left(M_{\max 1}-M_{\max 2}\right)$.

When $x=x_{t 2}, \rho_{x_{t 2}}=\rho_{\text {lim }}$, the boundary point $x_{t 2}$ of elastic-plastic deformation is

$$
x_{t 2}=-l_{0}+\left(\frac{2 \sigma_{s}}{E t}\right)^{n} \frac{k I_{p}\left(l_{0}-d_{2}\right)}{M_{\max }}
$$

When $x=-d_{2}$, the rotation angle of this particle can be obtained from (47)

$$
\alpha_{d}=\sin ^{-1}\left(\frac{d_{2}}{r_{p}+t / 2}\right)
$$

(2) The Springback Process. According to the unloading law, the radius of curvature at particle $x$ after springback is

$$
\begin{aligned}
& \frac{1}{\rho_{x}^{u}}=\left[\frac{M_{\max }\left(l_{0}+x\right)}{k I_{p}\left(l_{0}-d_{2}\right)}\right]^{1 / n}-\frac{M_{\max }}{E I} \frac{\left(l_{0}+x\right)}{\left(l_{0}-d_{2}\right)} \\
& x \in\left[-x_{t 2},-d_{2}\right]
\end{aligned}
$$


TABLE 1: Material properties of sheet metal.

\begin{tabular}{lcccc}
\hline Name & The yield strength & Coefficient of strength & Modulus of elasticity & Hardening index \\
& $\sigma_{s} / \mathrm{MPa}$ & $k / \mathrm{MPa}$ & $E / G P a$ & $n$ \\
\hline Q235b steel plate & 236.12 & 540.11 & 206.15 & 0.19 \\
\hline
\end{tabular}

Then, the rotation angle at particle $x \in\left[x_{t 2},-d_{2}\right]$ after unloading and springback is

$$
\begin{aligned}
& \alpha_{x}^{u}=\sin ^{-1} \phi_{2}(x) \\
& \phi_{2}(x)=-\frac{n}{n+1}\left[\frac{M_{\max }}{k I_{p}\left(l_{0}-d_{2}\right)}\right]^{1 / n} \\
& \cdot\left[\left(l_{0}-x\right)^{(n+1) / n}-\left(l_{0}-d_{2}\right)^{(n+1) / n}\right] \\
& +\frac{M_{\max }}{2 E I\left(l_{0}-d_{2}\right)}\left[\left(l_{0}-x\right)^{2}-\left(l_{0}-d_{2}\right)^{2}\right] \\
& \quad+d_{2}\left(\frac{1}{r_{p}+t / 2}-\frac{M_{\max }}{E I}\right)
\end{aligned}
$$

\section{Experimental Verification and Numerical Simulation}

5.1. Material Parameters and Experimental Equipment. Tensile test was carried out with Q235b steel plate. The yield strength $\sigma_{s}$, strength coefficient $k$, hardening index $n$, and elastic modulus $E$ of the material were obtained after analysis and treatment, as shown in Table 1.

The five-point bending test device is mainly composed of the following parts: electronic universal testing machine, bending mold, resistance strain gauge, data acquisition card, pressure sensor, and computer, shown in Figure 4. The model of the electronic universal testing machine is AG-100, the capacity is $100 \mathrm{kN}$, and the resolution is $0.01 \mathrm{~mm}$. The strain gauge is located on both sides of the bottom mold (Figure 4). The pressure sensor is located in the electronic universal testing machine. The electronic universal testing machine can collect the displacement and force of the upper die. The resistance strain gauge can collect the strain data of the bottom mold, by calibrating the bottom mold in advance, the relationship between deformation and force is obtained, and then the force of the bottom roll in bending process is obtained.

5.2. Establishment of Finite Element Model. Abaqus is one of the most advanced large-scale general-purpose nonlinear finite element analysis software. It has powerful calculation functions and extensive simulation performance. In this paper, Abaqus software is used to simulate loading and unloading process of five-point bending. The five-point bending process belongs to plane strain deformation, so this paper uses a two-dimensional model to simulate its bending process. In addition, because the boundary conditions and geometric models are simple, static implicit algorithms can be used to simulate the loading and unloading process. The plate parameters are the same as the experimental plate parameters. Define the die as a discrete rigid body. Set 3 elements on the thickness of the sheet. The element type is CPE4 (quadrilateral plane strain element), and the number of nodes is 1200 . Use the general contact to simulate contact conditions between the sheet and the mold. The upper roll die can only move in the y direction, and the lower roll die and the middle roll die are fixed. The y direction displacement of the sheet section element when the upper die is pressed $8 \mathrm{~mm}$ is as shown in Figure 5. The unit is millimeter.

5.3. Comparison of Theoretical Results, Numerical Simulation, and Experimental Results of Bending Force. Using the fivepoint bending theory and the same finite element geometry to calculate the bending force, compare and analyze the displacement of the upper roll die. The relevant calculation parameters are plate width $B=200 \mathrm{~mm}$, thickness $t=2 \mathrm{~mm}$, the yield strength $\sigma_{s}=236.12 \mathrm{MPa}$, coefficient of strength $k=540.11 \mathrm{MPa}$, hardening index $n=0.19$, modulus of elasticity $E=206.15 \mathrm{GPa}$, the radius of upper roll die $r_{p}=$ $12.5 \mathrm{~mm}$, the radius of bottom roll die $r_{d}=15 \mathrm{~mm}$, the radius of the lower roll die $r_{f}=20 \mathrm{~mm}$, the span between the lower roll die, and the bottom roll die $L_{3}=95 \mathrm{~mm}$. According to the experimental results in reference [24], the friction coefficient has little effect on the rebound angle. By simulating the whole process of FFX forming with explicit method, it is found that when the friction coefficient is 0.15 , the strip can enter the roll gap smoothly; thus, the friction coefficient in the theoretical calculation is 0.15 . Figure 6 shows the comparison between theoretical calculation value of bending force and experimental results.

From Figure 6 we can see that the bending force increases as the bending stroke increases. There is a certain error between the theoretical calculation result, numerical simulation, and the experimental result, and the error increases as the bending stroke increases. The maximum relative error is about $9.79 \%$. At the same time, it can be found that the results of theoretical analysis are generally smaller than the experimental results and numerical simulation. The reasons for the errors are mainly concentrated in two aspects. On the one hand, the derivation of theoretical calculation formula is carried out under some assumptions, such as material hardening model, tension-free assumption, the assumption that the neutral layer, and the geometric center line always coincide; on the other hand, there is also a certain deviation in testing. On the whole, the theoretical value and experimental value are basically consistent, and the trend of change is consistent.

5.4. Comparison of Theoretical Results, Numerical Simulation, and Experimental Results of Springback Angle. In order to 

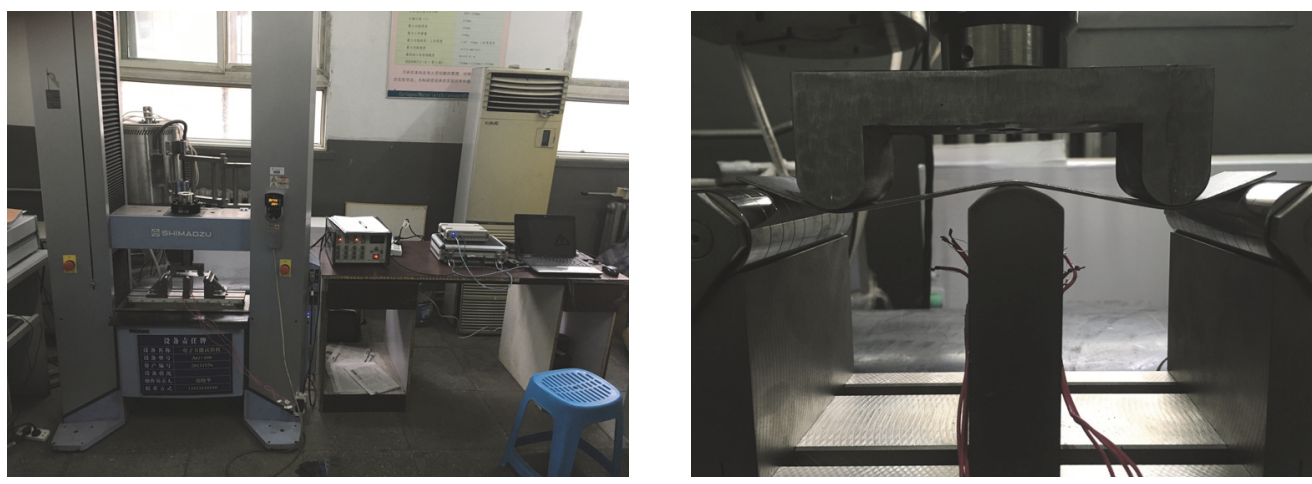

FIGURE 4: The test device of five-point bending.

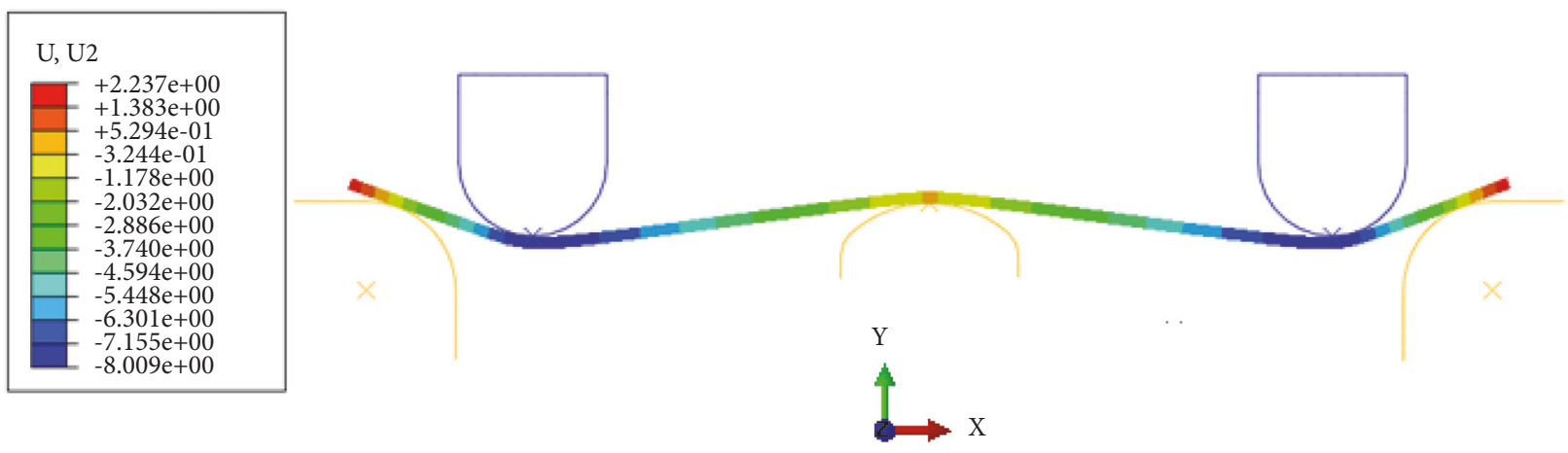

FIGURE 5: Finite element analysis displacement diagram.

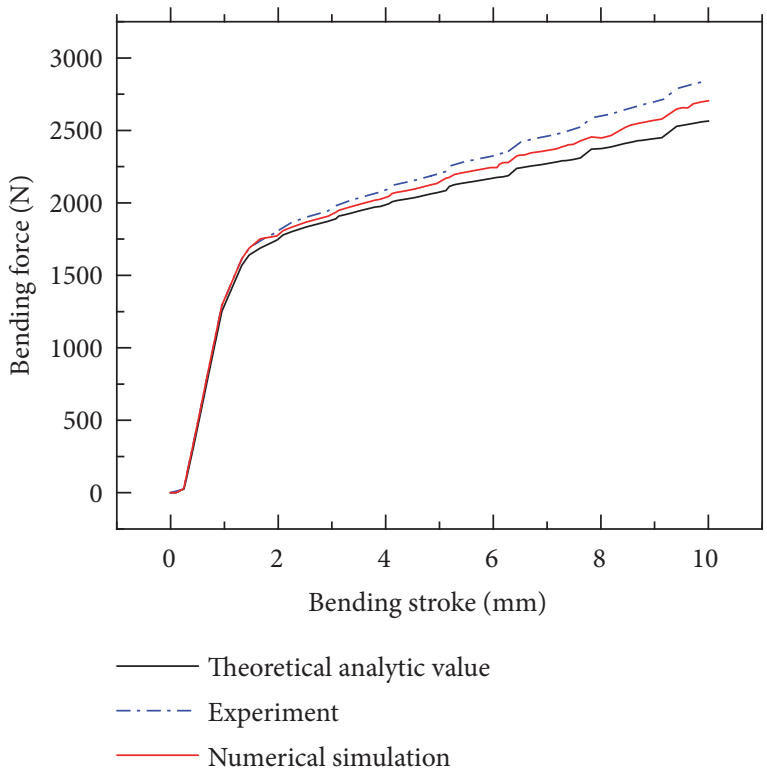

Figure 6: Comparison of bending force between the experimental results, numerical simulation, and theoretical analysis.

verify the correctness of the analytical results of springback theory, theoretical values of springback angle under different bending stroke were calculated according to the analytical

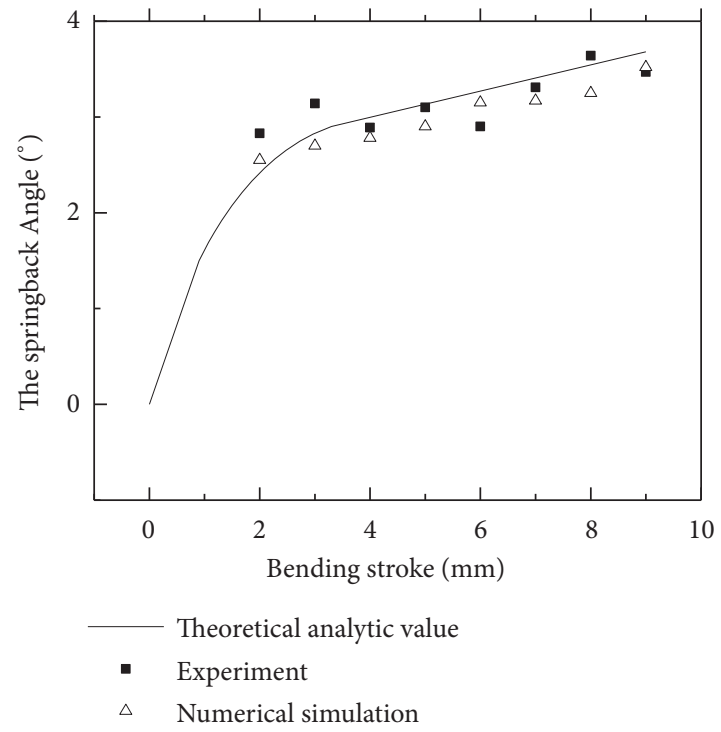

FIGURE 7: The comparison of springback angle between the theoretical results, numerical simulation, and the experimental results.

formula derived above and compared with numerical simulation and experimental results as shown in Figure 7.

From Figure 7 we can see that the springback angle increases with the increase of bending stroke, when the 
bending stroke is $8 \mathrm{~mm}$, the corresponding rebound angle is about $3.7^{\circ}$. At the same time, experimental and numerical simulation results show that the theoretical calculation of the rebound angle agrees well with the experimental results, the overall trend is the same, and the largest angular deviation is $0.43^{\circ}$. It shows that the five-point bending rebound model established in this paper is reasonable and reliable.

\section{Conclusion}

(1) According to the five-point bending characteristics of "W" antibending pass in FFX preforming of ERW pipe, under the premise of various assumptions, the five-point free bending model was obtained by simplifying the contact state model of roll bending process. The bending process is divided into two stages: fully elastic bending stage and elastic-plastic bending stage. At the same time, the mechanical model was established piecewise, and the mathematical expressions of bending curvature and turning angle of a particle on the strip under any state were derived.

(2) According to the theory of plane elastic-plastic bending, the formulas for calculating the curvature of particles on the steel plate after unloading were derived, and the formulas for calculating the bending angle and rebound angle after springback were obtained.

(3) The law of springback angle of five-point bending changing with stroke is revealed. At the same time, the springback angle of the theoretical maximum angular deviation is $0.43^{\circ}$. The experimental results show that the fivepoint bending model is reasonable, which provide theoretical support for the realization of setting model of FFX forming for roll bending process.

\section{Data Availability}

The data used to support the findings of this study are available from the corresponding author upon request.

\section{Conflicts of Interest}

The authors declare that they have no conflicts of interest.

\section{Acknowledgments}

This work was supported by National Key R\&D Program of China (grant number 2018YFB1308703), National Natural Science Foundation of China (grant number 51404160), and the Science and Technology Innovation Foundation of Higher Education of Shanxi Province (grant number 2016163).

\section{References}

[1] Z. Peng, "Development trend of straight welded pipe mills both at home and abroad," Steel Pipe, vol. 4, pp. 13-18, 1998.

[2] J. Jiang, D. Li, Y. Peng et al., "Research on strip deformation in the cage roll-forming process of ERW round pipes," Journal of
Materials Processing Technology, vol. 209, no. 10, pp. 4850-4856, 2009.

[3] L. E. Lindgren, "Roll forming," in Handbook of Manufacturing Engineering and Technology, vol. 13, pp. 285-307, 2016.

[4] Z. Y. Cai, Z. Sui, F. X. Cai et al., "Continuous flexible roll forming for three-dimensional surface part and the forming process control," The International Journal of Advanced Manufacturing Technology, vol. 66, no. 1-4, pp. 393-400, 2013.

[5] B. D. Joo, S. W. Han, S. G. R. Shin, and Y. H. Moon, "Flexible roll forming process design for variable cross-section profile," International Journal of Automotive Technology, vol. 16, no. 1, pp. 83-88, 2015.

[6] K. Mäntyjärvi, M. Merklein, and J. A. Karjalainen, "UHS steel formability in flexible roll forming," Key Engineering Materials, vol. 410-411, pp. 661-668, 2009.

[7] Z. Hu, M. Li, Z. Cai et al., "Continuous flexible forming of threedimensional surface parts using bendable rollers," Materials Science and Engineering: A Structural, vol. 499, no. 1-2, pp. 234237, 2009.

[8] S.-C. Heo, Y.-H. Seo, J.-W. Park, T.-W. Ku, J. Kim, and B.-S. Kang, "Application of flexible forming process to hull structure forming," Journal of Mechanical Science and Technology, vol. 24, no. 1, pp. 137-140, 2010.

[9] B. Abeyrathna, B. Rolfe, L. Pan, R. Ge, and M. Weiss, "Flexible roll forming of an automotive component with variable depth," Advances in Materials and Processing Technologies, vol. 2, no. 4, pp. 527-538, 2016.

[10] S. Hu, "Discussing technical innovation of national ERW pipe based on Japanese developments of welded pipe technology," Welded Pipe, vol. 29, no. 6, pp. 53-57, 2006.

[11] X. Ji, H. Zhang, and H. Ma, "Discussion on the development and research of the technology of large diameter HFW pipe in abroad and domestic," Welded Pipe, vol. 32, no. 8, pp. 10-15, 2009.

[12] M. Li, Y. Liu, S. Su, and G. Li, "Multi-point forming: a flexible manufacturing method for a 3-d surface sheet," Journal of Materials Processing Technology, vol. 87, no. 1-3, pp. 277-280, 1999.

[13] R. H. Wagoner, H. Lim, and M.-G. Lee, "Advanced issues in springback," International Journal of Plasticity, vol. 45, pp. 3-20, 2013.

[14] W. D. Carden, L. M. Geng, D. K. Matlock, and R. H. Wagoner, "Measurement of springback," International Journal of Mechanical Sciences, vol. 44, no. 1, pp. 79-101, 2002.

[15] L. Chen, "Finite element simulation of springback in sheet metal forming," Applied Mechanics and Materials, vol. 50-51, pp. 615618, 2011

[16] R. H. Wagoner and M. Li, "Simulation of springback: throughthickness integration," International Journal of Plasticity, vol. 23, no. 3, pp. 345-360, 2007.

[17] J. Zhao, J. Yin, R. Ma, and L. Ma, "Springback equation of small curvature plane bending," Science China Technological Sciences, vol. 54, no. 9, pp. 2386-2396, 2011.

[18] J. Zhao, R. Zhai, Z. Qian, and R. Ma, "A study on springback of profile plane stretch-bending in the loading method of pretension and moment," International Journal of Mechanical Sciences, vol. 75, no. 10, pp. 45-54, 2013.

[19] J. Zhao, P. Zhan, R. Ma, and R. Zhai, "Prediction and control of springback in setting round process for pipe-end of large pipe," International Journal of Pressure Vessels and Piping, vol. 116, no. 1, pp. 56-64, 2014. 
[20] C. Liu, T. Yue, and D. Li, "A springback prediction method for a cylindrical workpiece bent with the multi-point forming method," The International Journal of Advanced Manufacturing Technology, vol. 12, pp. 1-13, 2018.

[21] Z. Jun, L. Jian, Q. Xiaoyang, W. Gang, S. Honglei, and M. Rui, "Study on intelligent control technology for forming steel pipe of pipeline with JCO process," Science China Technological Sciences, vol. 54, no. 10, pp. 2754-2759, 2011.

[22] H. Sun, X. Song, R. Ma, and J. Zhao, "Theoretical analysis of JCO forming and springback for sheet metal four-point bending in manufacture of LSAW pipe," Journal of Mechanical Engineering, vol. 25, no. 2, pp. 257-262, 2014.

[23] Y. Li, J. Zhao, P. Zhan et al., "Theoretical analysis of sheet metal free bending and springback," Journal of Plastic Engineering, vol. 16, no. 4, pp. 1-6, 2009.

[24] J. Li, "Research on JCO intelligent control technology for forming the large diameter longitudinal-seam submerged Arc welded pipe with JCO process," Yanshan University, 2009. 


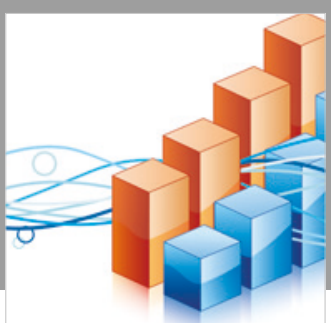

Advances in

Operations Research

\section{-n-m}
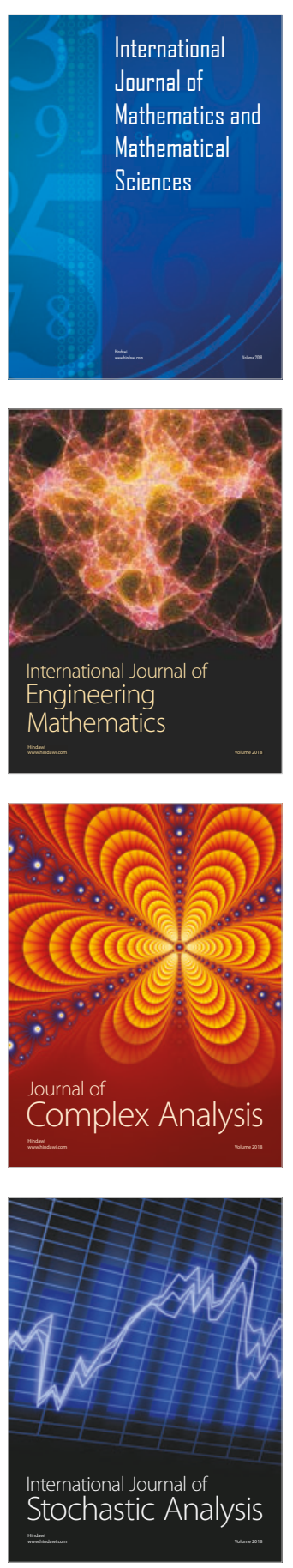
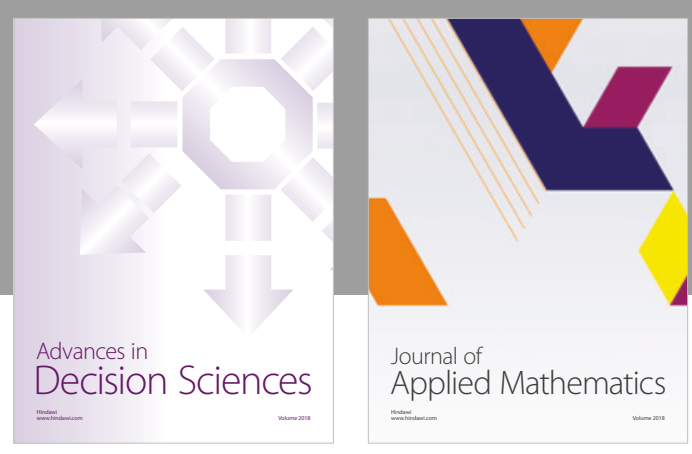

Journal of

Applied Mathematics
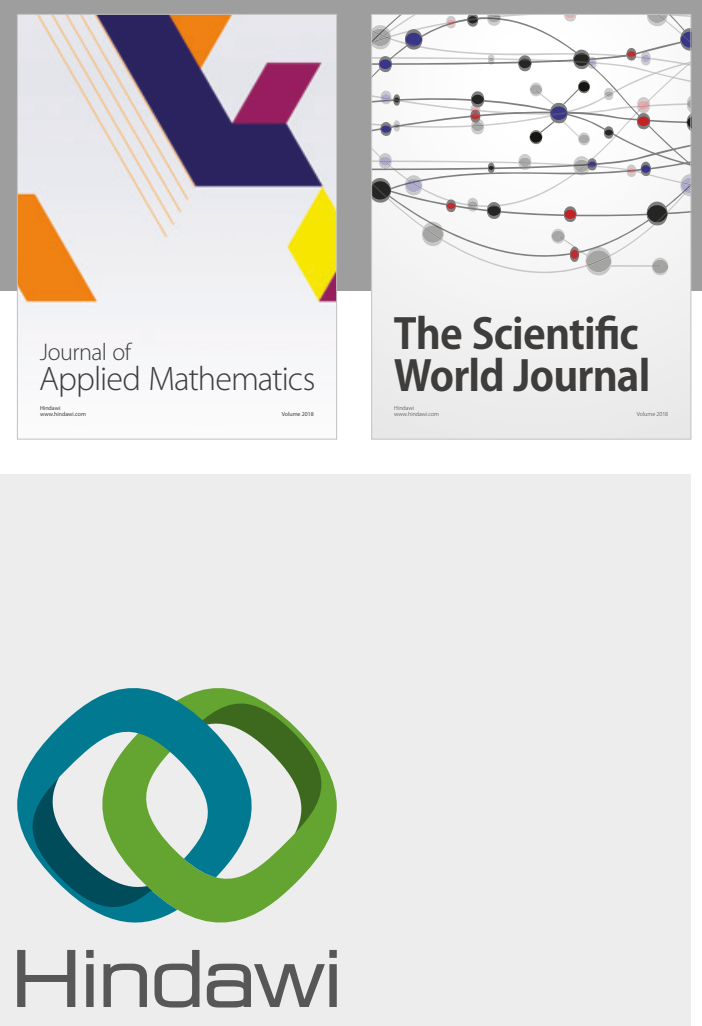

Submit your manuscripts at

www.hindawi.com

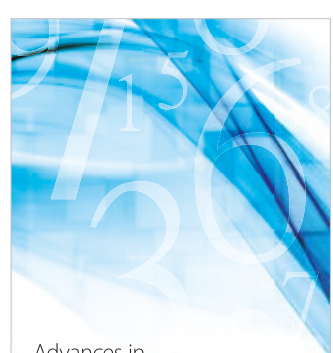

Advances in
Numerical Analysis
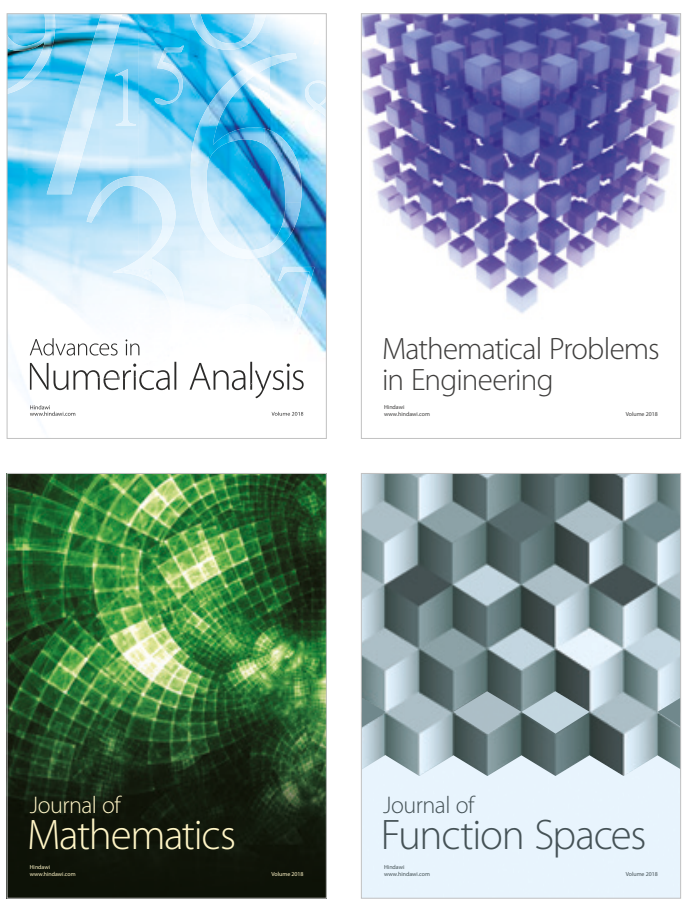

Mathematical Problems in Engineering

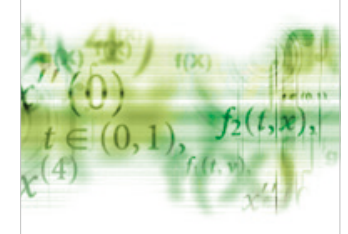

International Journal of

Differential Equations

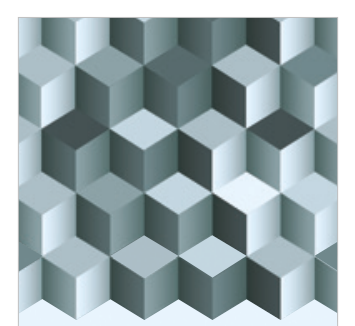

Journal of

Function Spaces
The Scientific

World Journal

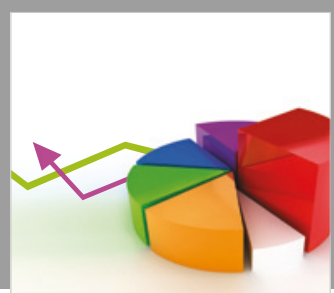

Journal of

Probability and Statistics
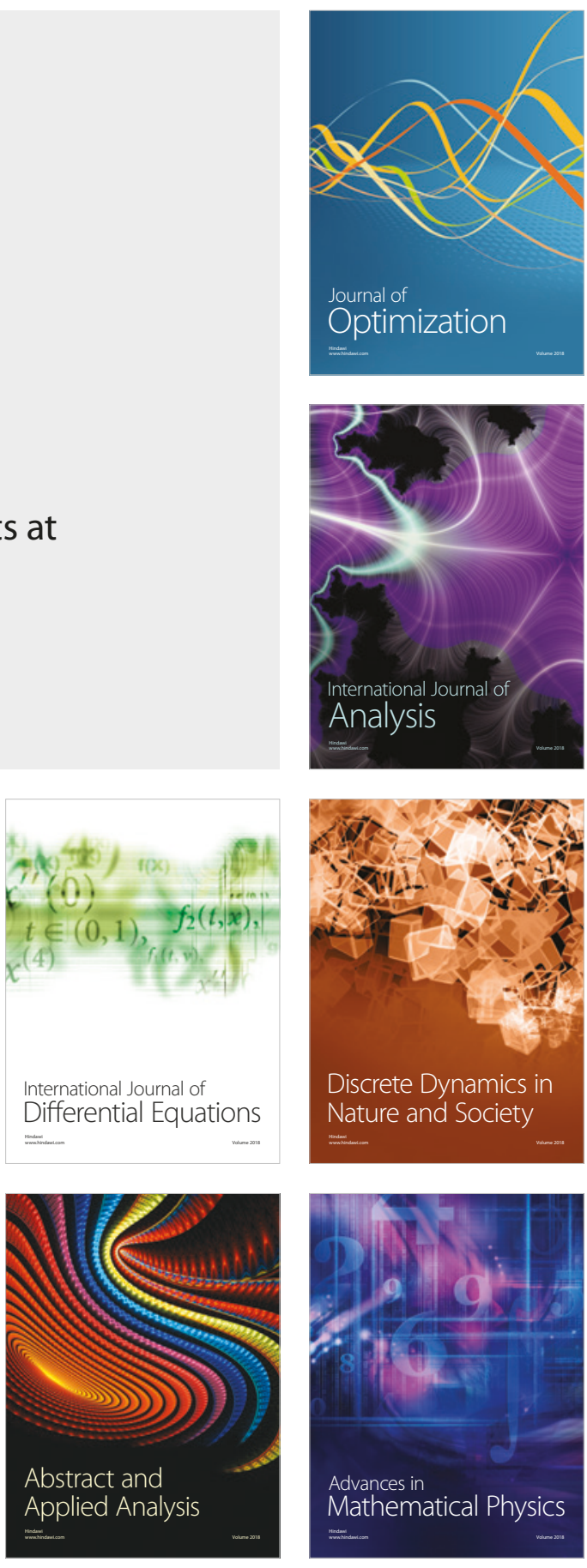